\title{
Beneficios y barreras del desplazamiento activo hacia el centro escolar: una revisión sistemática \\ Benefits and barriers of active commuting to the school center: a systematic review
}

Jesús Sáez Padilla, José Manuel Cantonero Cobos, Emilia Moreno Sánchez, Jorge Molina López, Inmaculada

Tornero Quiñones

Universidad de Huelva (España)

Resumen. La evidencia científica nos muestra cómo la falta de movimiento a lo largo del día puede tener efectos negativos en nuestra salud. Los desplazamientos activos al centro escolar permiten la adquisición de hábitos saludables siguiendo las recomendaciones de la Organización Mundial de la Salud. El objetivo de este artículo fue realizar una revisión sistemática sobre el estado del arte, en relación con el desplazamiento activo al centro escolar, las barreras existentes y sus beneficios a nivel físico, psicológico y motivacional en las diferentes etapas educativas. El diseño corresponde a una revisión de los estudios previamente publicados entre 2015 y 2020. Para la realización del trabajo se han utilizado las siguientes bases de datos: Web of Science, Pubmed y Scopus. Para la selección de los estudios, se identificaron sólo aquellos que coincidieron con los criterios preestablecidos. De los 25 resultados iniciales, se seleccionaron 13 artículos que cumplían los criterios de elegibilidad preestablecidos con anterioridad. Los resultados mostraron relación positiva entre el desplazamiento activo desde el hogar de residencia hasta el centro de estudios y beneficios asociados con la salud de los practicantes. En contraposición, se asociaron un número importante de barreras las cuales dificultan la elección de un transporte activo hacia las escuelas, institutos y/o universidades. Son necesarias más investigaciones y publicaciones de estudios que clarifiquen de forma específica y detallada los beneficios que reporta en la salud la realización de desplazamientos activos, así como los pasos a seguir en planes para minimizar las barreras que lo dificultan.

Palabras clave: actividad física, desplazamiento activo, sistema educativo, niños/as, jóvenes, beneficios.

\begin{abstract}
Scientific evidence shows us how a lack of movement throughout the day can have negative effects on our health. Active commuting to school enables the acquisition of healthy habits following the recommendations of the World Health Organization. The purpose of this article was to carry out a systematic review of the state of the art, in relation to active commuting to school, the existing obstacles and its physical, psychological, and motivational benefits at different educational stages. The design corresponds to a review of previous studies published between 2015 and 2020. The databases Web of Science, Pubmed and Scopus were used to carry out the study. For the selection of studies, only those that matched the preestablished standards were identified. From the 25 initial results, 13 articles were selected that met the pre-specified eligibility standards. The results showed a positive relationship between active commuting from home to school and associated health benefits for the practitioners. In contrast, a significant number of barriers were associated with the choice of active commuting to schools, high schools and/or universities. More research and published studies are needed to clarify specifically and detailed the health benefits of active commuting, as well as the steps to be taken in plans to minimize the barriers to active commuting.
\end{abstract}

Keywords: physical activity, active commuting, education system, children, youth, benefits.

\section{Introducción}

Cuando hablamos de actividad física (AF) nos estamos refiriendo al movimiento del cuerpo de forma voluntaria que conlleva un gasto de energía (Blair et al., 992). Sin embargo, entendemos por sedentarismo la ausencia de dicha AF e incluso realizando ejercicio a

Fecha recepción: 23-04-20. Fecha de aceptación: 29-07-21

Jesús Sáez Padilla

jesus.saez@dempc.uhu.es intensidades no adecuadas (Romero, 2009). Se ha evidenciado que las consecuencias de la falta de movimiento pueden tener efectos negativos en la salud, entre las que podemos distinguir pérdida de estimulación contráctil a nivel muscular, aumenta el riesgo de padecer algún tipo de cáncer (mama, colon, pulmón, etc.), diabetes tipo dos, enfermedades cardiovasculares (hipertensión, obesidad, síndrome metabólico, etc.) (Díez, 2017).

En la sociedad actual y, debido al ritmo frenético en el que vivimos, es cada vez más frecuente el uso del 
ocio pasivo tales como estar sentado frente a la pantalla del televisor, uso de videojuegos, ordenadores y aumento del transporte motorizado al lugar de trabajo (Owen et al., 2014; Van der Horst et al., 2007). Partiendo de esta premisa se hace indispensable concienciar a la población de la necesidad de practicar ejercicio físico seguro, fomentando un estilo de vida activo y saludable que mejorará nuestro estado físico y mental. De esta manera se hará frente al estrés y se reducirá el riesgo de padecer determinadas enfermedades y/o patologías.

La Organización Mundial de la Salud (OMS) (2010) estima que la falta de AF origina 1,9 millones de fallecimientos a nivel mundial. En este sentido, un reciente estudio expone como dicha inactividad física, tanto en zonas urbanas como rurales, ha incrementado en Europa desde 2002 hasta 2017 (Moreno-Llamaset et al., 2021). Por tanto, siguiendo las recomendaciones de la OMS, se sugiere que los niños/as y adolescentes acumulen 60 minutos diarios de AF moderada o vigorosa. No obstante, como hemos comentado anteriormente, es cada vez más frecuente la disminución de los niveles de AF en jóvenes que pueden llegar a continuar en la edad adulta. Por ello, la competencia motriz, como respuesta contextualizada, contribuye a que los jóvenes dispongan de un gran repertorio de acciones motrices (FortVanmeerhaeghe et al., 2017), de este modo se favorecerá las experiencias ricas en habilidades motrices que fomentará la práctica físico-deportiva en los jóvenes y con ello se reducirán los riesgos a padecer enfermedad por ausencia de esta. Janssen y LeBlanc (2010), señalan que una práctica moderada y regular de AF está asociada a numerosos beneficios en niños/as y adolescentes, como, por ejemplo: reducción de la presión arterial, bajo índice sobrepeso y obesidad, aumento densidad ósea, entre otras.

Para la superación de las barreras existentes, Ryan y Deci (2017) mencionan que la clave para que los jóvenes se involucren hacia la práctica de AF es la motivación, ya que es considerado como aquello que dirige el comportamiento humano. La Teoría de la Autodeterminación (TAD, Deci \& Ryan, 2000; Ryan \& Deci, 2017) es considerada como una de las teorías motivacionales con más éxito aplicadas al contexto físico-deportivo. La TAD, analiza cómo se desarrolla la personalidad estudiando el grado en el que el comportamiento humano es intencionado o auto-determinado (Deci \& Ryan, 1985). Dicha teoría, sostiene que el ser humano posee tres necesidades psicológicas básicas (NPBs) (competencia, autonomía y relación con los demás) a satisfacer en cualquier contexto.
Del mismo modo, la TAD, establece que la conducta del ser humano puede ser más o menos auto-determinada en función de su regulación motivacional. Los creadores de la teoría (Deci \& Ryan, 2000; Ryan \& Deci, 2000), mencionan que la satisfacción de las NPBs está relacionada con la motivación, ya que el aumento de la percepción de estas genera un incremento en la condición de motivación intrínseca y, por consiguiente, la frustración de estas producirá mayor motivación intrínseca o desmotivación. Por lo tanto, es vital emplear todos los recursos disponibles para aumentar la motivación de los educandos que beneficien la intención de seguir siendo físicamente activos una vez finalicen la etapa educativa, continuándose hasta la edad adulta y vejez.

Entre los recursos disponibles, con una evidencia científica contrastada, está el desplazamiento activo. Éste, se define como la acción de dirigirse al centro educativo y/o laboral por medio de transportes que conlleven un gasto energético, como andar o ir en bicicleta (Ruiz et al., 2015). En la actualidad, es cada vez más frecuente la investigación dentro del campo de la promoción del desplazamiento activo, ya que está relacionado con numerosos beneficios a nivel fisiológico y psicológico, así como una mayor adherencia a la práctica de AF (Molina-García et al., 2015; Villa-González et al., 2015; Chillón et al., 2017; Gálvez-Fernández et al., 2020). No obstante, existen numerosos condicionantes que dificultan dichos desplazamientos y, a consecuencia de ello, los niveles de AF se ven disminuidos de forma significativa entre el alumnado (Molina-García et al., 2016; Herrador Colmenero et al., 2018).

Con todo ello, el principal objetivo es realizar una revisión sistemática para comprender el estado actual del estudio entre los beneficios asociados a un desplazamiento activo en niños/as y jóvenes, así como las barreras existentes para dicho desplazamiento en el fomento de un estilo de vida activo y saludable.

\section{Método}

En este trabajo se incluyeron los principales métodos en una revisión sistemática, según la declaración PRISMA (Moher et al., 2015; Perestelo-Pérez, 2013; Urrutia \& Bonfill, 2010).

\section{Criterios de elegibilidad}

Para ser incluido en este trabajo de revisión, se requirió que los estudios incluyeran: a) realizado dentro del sistema educativo (educación primaria, secundaria 
o universitaria), c) evaluación de al menos una de estas variables: hábitos saludables, actividad física y/o transporte activo al colegio o instituto, e) publicados en inglés y castellano f) publicados en alguna de estas bases de datos: Web of Science, Pubmed y Scopus, g) publicación de los artículos entre los años 2015 hasta 2020, h) investigaciones realizadas en España.

\section{Fuentes de información}

La búsqueda se realizó en Web of Science, Scopus y Pubmed, desde 2015 hasta 2020. Se realizaron combinaciones de palabras claves en inglés: «Health» OR «Transportation» $\mathrm{OR}$ «Student» $\mathrm{OR}$ «Active transport» OR «Adolescent» OR «School» OR «Active Commuting» OR «Youths» OR «Physical Activity» OR «Kids» OR «Young people», siendo las más frecuentes; Physical activity, Active commuting, School. Además, se limitaron los estudios usando el operador: NOT «adults».

Selección de estudios y proceso de extracción de datos

Una vez realizadas las búsquedas se evaluó las bases de datos empleadas, se evaluó el título, el resumen y el método de cada artículo con el fin de identificar los artículos relacionados con la pregunta de investigación y descartar aquellos que no cumplían los criterios de elegibilidad mencionados anteriormente. Para la extracción de datos de los artículos seleccionados, se usó una plantilla previamente diseñada que incluía: (a) autor, y año de publicación; (b) datos de la muestra (tamaño, media de edad o franja de edad cuando el estudio no presentó edad media, y sexo); (c) principales resultados; (d) beneficios o barreras; y (e) base de datos.

\section{Resultados}

\section{Selección de los estudios}

En la figura 1 se muestra que, inicialmente, se identificaron 25 estudios en las diferentes bases de datos. A continuación, se eliminaron aquellos cuales no estuvieran en las bases de datos de Web of Science, Scopus o Pubmed. De éstos, 13 artículos cumplieron los criterios de elegibilidad y fueron incluidos en la revisión sistemática.

\section{Características de los estudios}

En la tabla 1 se muestran las características de los 13 estudios analizados que cumplieron con los criterios de inclusión. Todos ellos fueron publicados dentro del ran-

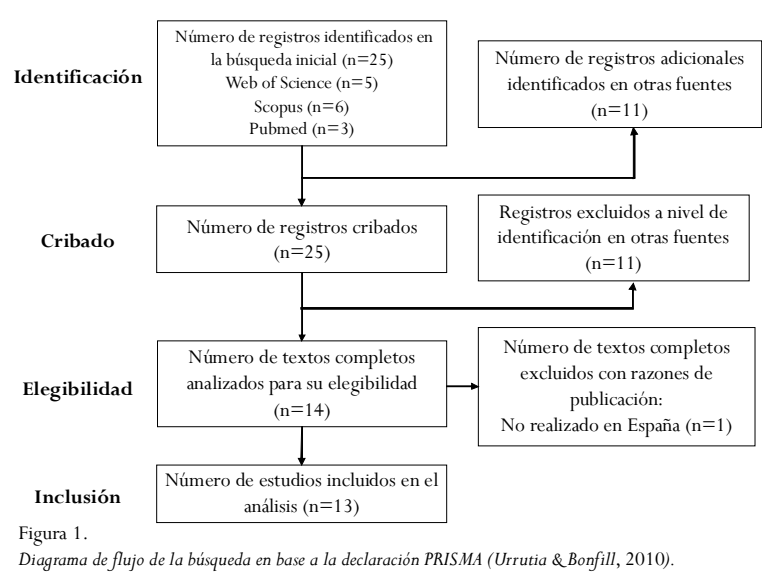

go de años entre 2015-2020 y fueron realizados en España. Se incluyeron 26796 participantes los cuales eran niños/as y adolescentes junto con 647 participantes los cuales eran padres/madres, haciendo un total de 27443 participantes. La muestra en los diferentes estudios varió desde $n=173 \mathrm{a} n=6979$. La edad media de los sujetos osciló entre 9.1 y 21.3 en niños/as y adolescentes; y de 43.2 en adultos (padres/madres).

Tabla 1.

Principales resultados obtenidos en la revisión realizada.

\section{Discusión}

El objetivo de esta revisión fue conocer el estado actual de estudio de las relaciones entre desplazamiento activo desde el hogar de residencia hasta el centro educativo (escuela, instituto y/o universidad) y viceversa, así como los posibles beneficios a nivel físico, psicológico y las posibles barreras existentes para la no realización de dicho desplazamiento en niños/as y/o adolescentes.

En lo referente a los beneficios físicos que el desplazamiento activo puede generar en la salud de los escolares que viajan desde su hogar de residencia hasta su centro de estudios son varios. Siguiendo a Molina-García et al. (2015), los usuarios del programa de acción de bicicleta pública gastaban, según el Índice Metabólico (MET), una equivalencia a 257 MET/Semana al desplazarse en bicicleta, por lo que había una pequeña reducción en el índice de masa corporal (IMC) contribuyendo así al control del peso corporal, en contraposición a lo que mencionan Gelabert, Muntaner-Mas y Palou (2019), destacan que un aumento de desplazamientos activos no parece mostrar una relación significativa con unos valores más favorables en los indicadores analizados (composición corporal y rendimiento académico). 
Tabla 1.

\begin{tabular}{|c|c|c|c|}
\hline $\begin{array}{c}\text { Autor (Año publ.) } \\
\text { [Beneficios/Barreras] }\end{array}$ & Muestra & Principales resultados & $\begin{array}{c}\text { Bases } \\
\text { de datos }\end{array}$ \\
\hline $\begin{array}{l}\text { Molina-García et al. } \\
\quad(2015) \\
\text { [Beneficios/Barreras] }\end{array}$ & $\begin{array}{c}\mathrm{N}=173 \\
\text { Hombres }=55 \\
\text { Mujeres }=117 \\
\text { Edad media }=21.3\end{array}$ & $\begin{array}{l}\text { Mostraron un aumento significativo (14.6\%) en la acción/etapa de mantenimiento del cambio. } \\
\text { No hubo cambio en la etapa de comportamiento cuando los estudiantes viajaban en moto/coche, siendo una barrera la } \\
\text { distancia de la vivienda al centro universitario o no tener estaciones de bicicleta a unos } 250 \mathrm{~m} \text { de su casa. } \\
\text { Los usuarios de SABP tienen una pequeña reducción del índice de masa corporal (IMC). }\end{array}$ & Pubmed \\
\hline $\begin{array}{l}\text { Ruiz-Ariza, de la Torre-Cruz, Redecillas- } \\
\text { Peiró y Martínez-López } \\
(2015) \\
\text { [Beneficios] }\end{array}$ & $\begin{array}{c}\mathrm{N}=1012 \\
\text { Hombres }=430 \\
\text { Mujeres }=582 \\
\text { Edad media }=14.42\end{array}$ & $\begin{array}{l}\text { Los/las adolescentes que emplean más de } 15 \text { minutos al día en desplazamiento activo tenían niveles más altos de felicidad } \\
\text { subjetiva y bienestar psicológico, así como niveles más bajos de angustia psicológica que los/las que emplean } 15 \text { o menos } \\
\text { minutos al día. } \\
\text { No se hallaron diferencias significativas en la imagen corporal entre los/las adolescentes menos y más activos/as. }\end{array}$ & Scopus \\
\hline $\begin{array}{l}\text { Villa-González et al. } \\
\text { (2015) } \\
\text { [Beneficios] }\end{array}$ & $\begin{array}{c}\mathrm{N}=723 \\
\text { Hombres }=494 \\
\text { Mujeres }=229 \\
\text { Edad media }=9.2\end{array}$ & $\begin{array}{l}\text { El transporte activo a la escuela se asoció con niveles más altos en la velocidad-agilidad y caf } \\
\text { inferior del cuerpo en niños y niñas, respectivamente que los niños/as que eran menos activos. }\end{array}$ & Pubmed \\
\hline $\begin{array}{l}\text { Molina-García et al. } \\
\qquad \text { (2016) } \\
\text { [Barreras] }\end{array}$ & $\begin{array}{c}\mathrm{N}=465 \\
\text { Hombres }=210 \\
\text { Mujeres }=255 \\
\text { Edad media }=15.4\end{array}$ & $\begin{array}{l}\text { Los resultados mostraron que la escala tenía índices de ajuste satisfactorios con dos factores: } \\
\text {-Un factor incluyó los items relativos a } \\
\text { ambiente y seguridad, y otro los ítems sobre planificación y aspectos psicosociales. } \\
\text {-El transporte activo se relacionó significativamente con la puntuación total de la escala de barreras, con las barreras } \\
\text { ambientales/seguridad y con las barreras de planificación/psicosociales. }\end{array}$ & Scopus \\
\hline $\begin{array}{l}\text { Chillón et al. } \\
\text { (2017) } \\
\text { [Beneficios] }\end{array}$ & $\begin{array}{c}\mathrm{N}=366 \\
\text { Hombres }=194 \\
\text { Mujeres }=172 \\
\text { Edad media }=9.3\end{array}$ & $\begin{array}{l}\text { Los niños/as que se desplazan con mayor frecuencia de forma activa al colegio tienen un menor nivel de estrés. } \\
\text { No hubo asociación entre desplazamiento activo con los niveles de felicidad subjetiva, bienestar subjetivo de tipo } \\
\text { cognitivo, afecto positivo y negativo. }\end{array}$ & $b$ of \\
\hline $\begin{array}{l}\text { Herrador-Colmenero, Villa-González y } \\
\text { Chillón } \\
(2017) \\
\text { [Barreras] }\end{array}$ & $\begin{array}{c}\mathrm{N}=652 \\
\text { Hombres }=339 \\
\text { Mujeres }=313 \\
\text { Edad media }=9.11\end{array}$ & $\begin{array}{l}\text { Los niños de 10-12 años eran más propensos a los viajes a la escuela sin compañía, más propensos a viajar de forma activa } \\
\text { y tenía una mejor percepción de seguridad que los niños más pequeños. } \\
\text { Los niños/as de } 10-12 \text { años de edad que viajaron activamente sin compañía tenía una mejor comprensión de las } \\
\text { cuestiones de seguridad que aquellos que lo hacían acompañados. }\end{array}$ & \\
\hline $\begin{array}{l}\text { Rodríguez-López et al. } \\
\text { (2017) } \\
\text { [Barreras] }\end{array}$ & $\begin{array}{c}\mathrm{N}=5998 \\
\text { Hombres }=3007 \\
\text { Mujeres }=2991 \\
\text { Edad media }=12.15\end{array}$ & $\begin{array}{l}\text { Alrededor del } 67 \% \text { de los niños } / \text { as y el } 60 \% \text { de los adolescentes se desplazan a la escuela de forma activa } \\
\text { (principalmente aminando). } \\
\text { El umbral de distancia para caminar a la escuela fue } 875 \mathrm{~m} \text { en los niños/as, y fue mayor entre participantes de zona } \\
\text { urbana }(1250 \mathrm{~m}) \text { que en participantes rurales }(675 \mathrm{~m}) \text {. } \\
\text { El umbral de distancia para caminar a la escuela entre adolescentes fue de } 1350 \mathrm{~m} \text {, siendo menor entre los de zona } \\
\text { urbana }(1350 \mathrm{~m}) \text { que en los participantes rurales }(1550 \mathrm{~m}) \text {. }\end{array}$ & \\
\hline $\begin{array}{c}\text { Chica-Olmo, Rodríguez-López y Chillón } \\
\text { (2018) } \\
\text { [Barreras] }\end{array}$ & $\begin{array}{l}\mathrm{N}=527 \\
\text { Edades comprendidas } \\
\text { entre } 12 \text { y } 18 \text { años. }\end{array}$ & $\begin{array}{l}\text { Los resultados indicaron que la (Euclidean) y las distancias a pie eran los mejores predictores de la decisión de ir a la } \\
\text { escuela de forma activa. } \\
\text { Además, el rango espacial de esta dependencia espacial es de aproximadamente } 1000 \mathrm{~m} \text { a } 1600 \mathrm{~m} \text {. }\end{array}$ & Scopus \\
\hline $\begin{array}{l}\text { Herrador-Colmenero et al. } \\
\text { (2018) } \\
\text { [Barreras] }\end{array}$ & $\begin{array}{c}\mathrm{N}=6979 \\
\text { Hombres }=3531 \\
\text { Mujeres }=3448 \\
\text { Edad media }=13.53\end{array}$ & $\begin{array}{l}\text { En invierno, niños/as (de } 7 \text { a } 11 \text { años de edad) fueron menos propensos a elegir un modo activo de desplazamiento a la } \\
\text { escuela. } \\
\text { En primavera, adolescentes ( } 12-18 \text { años) eran más propensos a elegir un modo activo de desplazamiento a la escuela. } \\
\text { Con temperaturas medias más altas, adolescentes eran más propensos a elegir un modo activo. }\end{array}$ & Pubmed \\
\hline $\begin{array}{l}\text { Huertas-Delgado, Molina-García, Van } \\
\text { Dyckc y Chillón } \\
\text { (2018) } \\
\text { [Barreras] }\end{array}$ & $\begin{array}{c}\mathrm{N}=647 \\
\text { (padres/madres) } \\
\text { Hombres }=472 \\
\text { Mujeres }=175 \\
\text { Edad media }=43.2 \\
\end{array}$ & $\begin{array}{l}\text { El cuestionario mostró una buena consistencia interna para el cuestionario en general y para las tres escalas. } \\
\text { Los valores de correlación intra-clase fueron moderadas. } \\
\text { El cuestionario general y las escalas de barreras generales y caminar mostraron una moderada validez para predecir } \\
\text { modos activos de los desplazamientos, así como 'distancia' y 'conveniencia caminar' }\end{array}$ & Scopus \\
\hline $\begin{array}{l}\text { Muntaner-Mas, Herrador-Colmenero, } \\
\text { Borràs y Chillón } \\
(2018) \\
\text { [Beneficios] }\end{array}$ & $\begin{array}{c}\mathrm{N}=2518 \\
\text { Hombres }=1214 \\
\text { Mujeres }=1304 \\
\text { Edad media }=13.0\end{array}$ & $\begin{array}{l}\text { Los modelos multinivel mostraron asociaciones entre aptitud cardiorrespiratoria y actividad física (AF) en los } \\
\text { adolescentes, pero no en niños. } \\
\text { Sin embargo, la aptitud cardiorrespiratoria no se asoció con el modo de desplazamiento a la escuela ya sea en niños o } \\
\text { adolescentes. } \\
\text { Los adolescentes que cumplieron con las recomendaciones de AF tuvieron mejor aptitud cardiorrespiratoria en } \\
\text { comparación con aquellos que no lo hicieron, independientemente de cómo se desplazaron a la escuela. }\end{array}$ & Scopus \\
\hline $\begin{array}{l}\text { Ferri-García, Fernández-Luna, Rodríguez- } \\
\text { López y Chillón } \\
\text { (2019) } \\
\text { [Barreras] }\end{array}$ & $\begin{array}{c}\mathrm{N}=6979 \\
\text { Hombres }= \\
\text { Mujeres }= \\
\text { Edad media }=13.2\end{array}$ & $\begin{array}{l}\text { Los resultados demostraron que el transporte activo puede estar influenciado por varios factores. El principal factor } \\
\text { parece ser la distancia a la escuela; un umbral de entre } 1100 \mathrm{~m} \text { y } 1600 \mathrm{~m} \text {. fue la distancia a la que los estudiantes } \\
\text { cambiaron de un activo a un modo pasivo de los desplazamientos. } \\
\text { También se detectó que las condiciones meteorológicas específicas podrían influir en transporte activo de alguna manera. } \\
\text { Por último, algunos factores tales como edad más avanzada y que viven en zonas urbanas (en contraposición a las zonas } \\
\text { rurales) se asociaron con los modos activos de los desplazamientos. }\end{array}$ & \\
\hline $\begin{array}{l}\text { Burgueño, González-Cutre, Sevil-Serrano, } \\
\text { Herrador-Colmenero, Segura-Díaz, } \\
\text { Medina-Casaubón y Chillón } \\
\text { (2019) } \\
\text { [Barreras }]\end{array}$ & $\begin{array}{l}\mathrm{N}=404 \\
\text { Hombres }=197 \text { Mujeres }= \\
207 \\
\text { Edad media }=14.25\end{array}$ & $\begin{array}{l}\text { La motivación de los adolescentes tiene un papel a considerar cuando se explican los procesos que implican transporte } \\
\text { activo a la escuela (TAE), y su medición a través de este instrumento debe ser considerada en conjunto otras variables } \\
\text { que tradicionalmente se han utilizado para explicar el comportamiento de TAE (por ejemplo, la distancia desde su casa a } \\
\text { la escuela, la percepción de la seguridad vial, barreras para transporte activo, el modo de conmutar a su lugar de trabajo) } \\
\text { de los padres. }\end{array}$ & Scopus \\
\hline
\end{tabular}

Villa-González et al. (2015), apuntan que el transporte activo hacia la escuela (principalmente andando, aunque también en bicicleta) encuentran asociaciones relacionada con la condición física y la salud, concretamente muestran que el transporte activo hacia la escuela se asocia con puntuaciones más elevadas en pruebas de velocidad-agilidad en niños y puntuaciones más altas en pruebas de salto de longitud en niñas. Por ello, estos autores demuestran que los niños/as que realizaban un transporte activo, tenían más velocidad-agilidad y capacidad muscular en el tren inferior, respectivamente, que los escolares menos activos en sus desplazamientos al centro de estudios.

Asimismo, Muntaner-Mas et al. (2018), aportan asociaciones entre la aptitud cardiorrespiratoria y la $\mathrm{AF}$ en adolescentes, no siendo así en niños/as. Del mismo modo, la aptitud cardiorrespiratoria no se asocia con el estilo de desplazamiento a la escuela ya sea en niños/as o adolescentes. No obstante, dichos autores mencionan que, aquellos que cumplieron las recomendaciones de AF diaria, obtuvieron mejor aptitud cardiorrespiratoria con respecto a los que no las siguieron, independientemente del tipo de desplazamiento hacia y/o desde la escuela. Por su parte, Ruiz-Ariza et al. (2015), indica que aquellos estudiantes que emplean más de 15 minutos al día en desplazamiento activo no presentan diferencias significativas en lo referente a la imagen corporal entre los adolescentes más y menos activos.

Además de los beneficios a nivel físico mencionados anteriormente, existen estudios que indican beneficios 
a nivel psicológico y motivacionales con respecto a la realización de un desplazamiento activo. Ruiz-Ariza et al. (2015), evidencian que los/las adolescentes que empleaban mayor tiempo al día en cuanto al desplazamiento activo, tenían mejores niveles de felicidad subjetiva y bienestar, así como una menor angustia psicológica independientemente de la edad, sexo o índice de masa corporal. Muestran también como aquellos/as adolescentes que emplean un tiempo igual o superior a 15 minutos al día en el desplazamiento activo, tienen mayor felicidad y bienestar, así como una mejor angustia que aquellos que no emplean dicho tiempo.

Sin embargo, Chillón et al. (2014), indican que el desplazamiento activo no se asocia con los niveles de felicidad subjetiva, bienestar subjetivo de tipo cognitivo, afecto positivo y afecto negativo en el grupo de escolares estudiado. No obstante, sí que se asocia el desplazamiento activo con el estrés, ajustando por sexo, edad y nivel de estudios de la madre, aquellos niños/as que se desplazan con mayor frecuencia activamente al colegio presentan un menor nivel de estrés.

Por su parte, Burgueño et al. (2019) añaden el aspecto motivacional a considerar cuando se explica el transporte activo a la escuela, así como su medición a través de un instrumento adecuado, debe ser considerada en conjunto otras variables que tradicionalmente se han utilizado para explicar el comportamiento. Muestran también como la motivación intrínseca, motivación integrada y regulación identificada, influyen de forma positiva en el comportamiento del transporte activo hacia la escuela.

Además de los beneficios a nivel físico y psicológico, así como aspectos motivacionales, existen estudios revisados los cuales analizan las barreras percibidas a la hora de realizar un desplazamiento activo desde el hogar hacia el centro educativo y viceversa. Molina-García et al. (2015), indican como principal barrera la distancia desde su hogar hacia la universidad ( $5 \mathrm{~km}$ ), así como no tener estaciones de bicicletas de uso público a unos 250 $\mathrm{m}$ de su casa. Del mismo modo, aquellos/as que si utilizaron el desplazamiento activo hacia la universidad (bicicleta), percibieron barreras en cuanto a la seguridad vial.

Rodríguez-López et al. (2017), indican que el umbral de distancia para caminar a la escuela fue de $875 \mathrm{~m}$ en niños/as, siendo mayor entre los participantes de zona urbana $(1250 \mathrm{~m})$ que los participantes de zonas rurales $(675 \mathrm{~m})$. Asimismo, el umbral para los adolescentes fue de $1350 \mathrm{~m}$, siendo menor en la zona urbana (1350 m) que los participantes de las zonas rurales (1550 m). Por su parte, Chica-Olmo et al. (2018) y FerriGarcía et al. (2020), indican el rango espacial entre el hogar y la escuela de entre 1000 m y 1600 m entre escolares con edades comprendidas entre 12 y 18 años.

Por su parte, Molina-García et al. (2016), muestran que las dificultades existentes en el transporte activo están relacionadas con las barreras ambientales, como son las condiciones meteorológicas, las barreras de seguridad junto a la falta de carril bici, cruces peligrosos o la no existencia de lugares para dejar la bicicleta con seguridad. En cuanto a las barreras de planificación y psicosociales, resaltamos las siguientes: «no se considera guay ir andando o en bicicleta al centro universitario, voy cargado con demasiadas cosas, no disfruto, demasiadas cuestas, etc.».

Herrador-Colmenero et al. (2018), indican barreras percibidas a nivel de estaciones y meteorología, siendo en invierno la estación menos propensa para elegir un modo de desplazamiento activo hacia la escuela en niños/as entre siete y 11 años. Del mismo modo, dichos autores indican que, en adolescentes, la estación primaveral era la más propensa para realizar un desplazamiento activo hacia el lugar de estudios, concluyendo que todos los participantes son más propensos a elegir un modo de desplazamiento activo con temperaturas medias más elevadas.

Por último, en cuanto a barreras, Huertas-Delgado et al. (2019), indican que los padres/madres perciben barreras para que sus hijos/as empleen un desplazamiento activo hacia la escuela como la no existencia de carril bici en su localidad, peso de la mochila a transportar, ausencia de otros niños/as en bicicleta, falta de vigilancia policial y poca seguridad en intersecciones. Asimismo, Herrador-Colmenero et al. (2017), resaltan que aquellos niños/as de entre 10 y 12 años de edad que se desplazaron activamente sin compañía tuvieron una mejor comprensión y percepción de la seguridad que aquellos/as que lo hacían acompañados.

\section{Conclusiones}

Los resultados de los artículos de investigación incluidos en esta revisión muestran una relación positiva entre el desplazamiento activo desde el hogar de residencia hasta el centro de estudios y beneficios físicos, psicológicos y motivacionales de los practicantes. En cuanto a los beneficios físicos que el desplazamiento activo puede generar en la salud de los escolares que viajan desde su hogar de residencia hasta su centro de estudios son: reducción en el índice de masa corporal (con- 
trol/ajuste del peso corporal), mejora en puntuaciones de pruebas de velocidad-agilidad y aumento de la capacidad muscular del tren inferior y mejor aptitud cardiorrespiratoria. En relación con los beneficios motivacionales y psicológicos, éstos son: mejor nivel de felicidad subjetiva y bienestar, menor angustia psicológica, menor nivel de estrés e influencia en la motivación intrínseca, integrada y regulación identificada.

En contraposición, se asocian un número importante de barreras percibidas, tales como; la distancia desde el hogar hacia el centro escolar, no tener estaciones de bicicletas de uso público cerca de sus viviendas, poca seguridad vial y/o ausencia de carril bici y barreras ambientales y/o meteorológicas, las cuales dificultan la elección de un transporte activo hacia las escuelas, institutos y/o universidades.

Una de las posibles limitaciones que puede tener este estudio, es la de haber realizado únicamente una revisión de aquellos estudios que realizaron revisiones $\mathrm{y} / \mathrm{o}$ intervenciones a nivel nacional. Por lo que una idea interesante a llevar a cabo en el futuro sería ampliar la revisión a nivel internacional con el objetivo de constatar si existe una homogeneidad entre los beneficios y barreras existentes a la hora de elegir un desplazamiento activo hacia el centro educativo.

Cabe destacar que cada vez es más frecuente, desde las instituciones y políticas públicas y/o privadas, el fomento de un desplazamiento activo. Por este motivo, revisiones de este tipo facilitan a dichas entes conocer el estado de la cuestión y aplicar estrategias, en función de los objetivos planteados, para que los escolares y adolescentes elijan un desplazamiento activo hacia el centro escolar y, de este modo, fomentar un estilo de vida activo y saludable que se prolongue en el tiempo y minimice el riesgo de padecer determinadas enfermedades y/o patologías.

En definitiva, son necesarias más investigaciones y publicaciones de estudios que clarifiquen de forma específica y detallada los beneficios que reporta en la salud la realización de desplazamientos activos, así como los pasos a seguir en planes para minimizar las barreras que lo dificultan aportando soluciones que incrementen el uso del transporte activo.

\section{Referencias}

Blair, S., Kohl, H., Gordon, N., \& Paffenbarger Jr. (1992). How much physical activity is good for health? Annual review of public health, 13(1), 99-126. https://doi.org/10.1146/ annurev.pu.13.050192.000531
Burgueño, R., González-Cutre, D., Sevil-Serrano, J., HerradorColmenero, M., Segura-Díaz, J., Medina-Casaubón, J., \& Chillón, P. (2019). Understanding the motivational processes involved in adolescents' active commuting behaviour: Development and validation of the Behavioural Regulation in Active Commuting to and from School (BR-ACS) Questionnaire. Transportation research part F: traffic psychology and behaviour, 62, 615-625. https://doi.org/10.1016/ j.trf.2019.02.016

Chica-Olmo, J., Rodríguez-López, C., \& Chillón, P. (2018). Effect of distance from home to school and spatial dependence between homes on mode of commuting to school. Journal of transport geography, 72, 1-12. https://doi.org/10.1016/ j.jtrangeo.2018.07.013

Chillon, P., Villén-Contreras, R., Pulido-Martos, M., \& Ruiz, J. (2017).Active commuting to school, positive health and stress in Spanish children. Sport TK: revista euroamericana de ciencias del deporte, 6(1), 117-124.

Deci, E.L., \& Ryan, R.M.(1985).The general causality orientations scale: Self-determination in personality. Journal of Research in Personality, 19(2), 109-134. https://doi.org/10.1016/00926566(85)90023-6

Deci, E. L., \& Ryan, R. M. (2000). The «what» and «why» of goal pursuits: human needs and the self-determination of behaviour. Psychological Inquiry, 11(4), 227-268.

http:/ / www.jstor.org/stable/1449618

Díez, C. (2017). Inactividad física y sedentarismo en la población española. Revista de investigación y educación en ciencias de la salud, 2(1), 41-48. https://doi.org/10.37536/ RIECS.2017.2.1.18

Ferri-García, R., Fernández-Luna, J., Rodríguez-López, C., \& Chillón, P. (2020). Data mining techniques to analyze the factors influencing active commuting to school. International Journal of SustainableTransportation, 14(4), 308-323.https://doi.org/ 10.1080/15568318.2018.1547465

Fort-Vanmeerhaeghe, A., Román, B., \& Font-Lladó, R. (2017). ¿Por qué es importante desarrollar la competencia motriz en la infancia y la adolescencia? Base para un estilo de vida saludable. Apunts: Medicina de l'esport, 52(195), 103-112.https:/ / doi.org/10.1016/j.apunts.2016.11.001

Gálvez-Fernández,P., Saucedo-Araujo, R. G., Campos-Garzón, P., Aranda-Balboa, M. J., Molina-Soberanes, D., Segura-Díaz, J. M., .. \& \& Chillón, P. (2020). El desplazamiento activo al centro educativo e indicadores de salud asociados: protocolo de evaluación del estudio PACO «Pedalea y Anda al Colegio» y su aplicación en educación secundaria. Retos, 39, 649-657. https:/ /doi.org/10.47197/retos.v0i39.80906

Gelabert Carulla, J., Muntaner-Mas, A., \& Palou, P. (2019). Influencia del desplazamiento activo al colegio sobre la composición corporal y el rendimiento académico en escolares de 10-12 años. Retos, 36, 376-383. https: / / doi.org/10.47197/ retos.v36i36.68166

Herrador Colmenero, M., Harrison, F., Villa González, E., RodríguezLópez, C., Ortega, F., Ruiz, J., ... Chillón,P. (2018). 
Longitudinal associations between weather, season, and mode of commuting to school amongst Spanish youths. Scandinavian journal of medicine \& science in sports, 28(12), 2677-2685. https: / /doi.org/10.1111/sms.13268

Herrador Colmenero, M.,Villa González, E., \& Chillón, P. (2017). Children who commute to school unaccompanied have greater autonomy and perceptions of safety. Acta Pediátrica, 106(12), 2042-2047. https://doi.org/10.1111/ apa. 14047

Huertas-Delgado, F., Molina-García, J.,Van Dyck, D., \& Chillón, P. (2019). A questionnaire to assess parental perception of barriers towards active commuting to school (PABACS): Reliability and validity. Journal of Transport \& Health, 12,97-104. https://doi.org/10.1016/j.jth.2018.12.004

Janssen, I., \& LeBlanc, A. (2010). Systematic review of the health benefits of physical activity and fitness in schoolaged children and youth. International Journal of Behavioral Nutrition and Physical Activity, 7(1), 40. doi: 10.1186/1479-5868-7-40

Moher, D., Shamseer, L., Clarke, M., Ghersi, D., Liberati, A., Petticrew, M., ... .Stewart, L. (2015). Preferred reportingitems for systematic review and meta-analysis protocols (PRISMAP) 2015 statement. Systematic Reviews, 4(1), 1.https://doi.org/ 10.1186/2046-4053-4-1

Molina-García, J., Castillo, I., Queralt, A., \& Sallis, J. (2015). Bicycling to university: evaluation of a bicycle-sharing program in Spain. Health promotion international, 30(2), 350-358. https:/ /doi.org/10.1093/heapro/dat045

Molina-García, J., Queralt,A., Estevan, I., Álvarez, O., \& Castillo, I. (2016). Barreras percibidas en el desplazamiento activo al centro educativo: fiabilidad y validez de una escala. Gaceta Sanitaria, 30(6), 426-431.http://dx.doi.org/10.1016/ j.gaceta.2016.05.006

Moreno-Llamas, A., García-Mayor, J., \& De la Cruz-Sánchez, E. (2021). Urban-rural differences in trajectoriesofphysicalactivity in Europefrom 2002 to 2017. Health\& Place, 69, 102570. https://doi.org/10.1016/j.healthplace.2021.102570

Muntaner-Mas, A., Herrador-Colmenero, M., Borràs, P., \& Chillón, P. (2018). Physical activity, but not active commuting to school, is associated with cardiorespiratory fitness levels in young people. Journal of Transport \& Health, 10,297-303.https:/ /doi.org/10.1016/j.jth.2018.05.004

ORDEN de 14 de julio de 2016, por la que se desarrolla el currículo correspondiente a la Educación Secundaria Obligatoria en la Comunidad Autónoma de Andalucía, se regulan determinados aspectos de la atención a la diversidad y se establece la ordenación de la evaluación del proceso de aprendizaje del alumnado. Consejería de Educación. Boletín Oficial de la Junta de Andalucía. Sevilla, 28 de junio de 2016, núm. 144, pp. 108-396.

Organización Mundial de la Salud. (2010) Recomendaciones mundiales sobre actividad fisica para la salud. Ginebra. Organización Mundial de la Salud

Owen, N., Salmon, J., Koohsari, M., Turrell, G., \& Giles-Corti, B. (2014). Sedentary behaviour and health: mapping environmental and social contexts to underpin chronic disease prevention. British Journal of Sports Medicine, 48(3), 174-177. http://dx.doi.org/10.1136/bjsports-2013-093107

Perestelo-Pérez, L. (2013). Standards on how to develop and report systematic reviews in Psychology and Health. International Journal of Clinical and Health Psychology, 13(1), 4957.

Rodríguez-López, C., Salas-Fariña, Z., Villa-González, E., BorgesCosic, M., Herrador-Colmenero, M., Medina-Casaubón, J., ...Chillón, P. (2017). The threshold distance associated with walking from home to school. Health Education \& Behavior, 44(6), 857-866.https://doi.org/10.1177/ 1090198116688429

Romero, T. (2009). Hacia una definición de Sedentarismo. Revista chilena de cardiología, 28(4), 409-413. http://dx.doi.org/ 10.4067/S0718-85602009000300014

Ruiz, A., García, F., Latorre, P, \& Martínez, E. (2015). Niveles de desplazamiento activo en jóvenes de 12-16 años. Un estudio de la provincia de Jaén. EmásF: revista digital de educación física, (34), 71-79.

Ruiz-Ariza, A., de la Torre-Cruz, M., Manzano-Suárez. S., \& Martínez-López, E. (2017). El desplazamiento activo al Centro educativo influye en el rendimiento académico de las adolescentes españolas. Retos: nuevas tendencias en educación fisica, deporte y recreación, (32), 39-43. https://www.redalyc.org/ articulo.oa?id $=3457 / 345751100008$

Ruiz-Ariza, A., de la Torre-Cruz, M., Redecillas-Peiró, M., \& Martínez-López, E. (2015). Influencia del desplazamiento activo sobre la felicidad, el bienestar, la angustia psicológica y la imagen corporal en adolescentes. Gaceta Sanitaria, 29(6), 454457.https://doi.org/10.1016/j.gaceta.2015.06.002

Ryan, R. M., \& Deci, E. L. (2000). Self-determination theory and the facilitation of intrinsic motivation, social development and well-being. American Psychologist, 55(1), 68-78. https:// doi.org/10.1037//0003-066x.55.1.68

Ryan, R. M., \& Deci, E. L. (2017). Self-Determination Theory. Basic Psychological Needs in Motivation, Development, andWelness. New York, NY, USA: Guilford Publications.

Urrutia, G., \& Bonfill, X. (2010). PRISMA declaration: a proposal to improve the publication of systematic reviews and metaanalyses. Medicina Clínica, 135(11), 507-511. https:// doi.org/10.1016/j.medcli.2010.01.015

Van der Horst, K., Paw, M., Twisk, J., \&Van Mechelen,W. (2007). A brief review on correlates of physical activity and sedentariness in youth. Medicine and Science in Sports and Exercise, 39(8), 1241 -1250. 10.1249/mss.0b013e318059bf35

Villa-González, E., Ruiz, J., \& Chillón, P. (2015). Associations between active commuting to school and health-related physical fitness in Spanish school-aged children: a crosssectional study. International journal of environmental research and public health, 12(9), 10362-10373. https://doi.org/ 10.3390/ijerph120910362 